\title{
Development of a waxy gene real-time PCR assay for the quantification of sorghum waxy grain in mixed cereal products
}

Jaemin Cho ${ }^{*}$, Taewook Jung, Jungin Kim, Seokbo Song, Jeeyeon Ko, Koansik Woo, Jaesaeng Lee, Myeongeun Choe and Inseok Oh

\begin{abstract}
Background: Waxy-grain sorghum is used in most of the commercial cereal products in Korea. Worldwide, three waxy mutant alleles have been identified in the sorghum germplasm, and DNA markers for these alleles have been developed to identify the waxy genotype. However, that detection method cannot be used to determine the proportion of waxy content in samples containing both waxy and non-waxy sorghum. This study developed an assay that can be used to detect and quantify the waxy content of mixed cereal samples.

Results: All Korean waxy-grain sorghum used in this study contained the $w x^{a}$ allele, and one $w x^{a}$ allele-containing individual was also heterozygous for the $w x^{c}$ allele. No individuals possessed the $w x^{b}$ allele. The genotyping results were confirmed by iodine staining and amylose content analysis. Based on the sequence of the $w x^{a}$ allele, three different types of primers ( $w x^{a}$ allele-specific, non-waxy allele-specific, and nonspecific) were designed for a quantitative real-time PCR (qPCR) assay; the primers were evaluated for qPCR using the following criteria: analytical specificity, sensitivity and repeatability. Use of this qPCR assay to analyze mixed cereal products demonstrated that it could accurately detect the waxy content of samples containing both waxy and non-waxy sorghum.

Conclusions: We developed a qPCR assay to identify and quantify the waxy content of mixed waxy and non-waxy sorghum samples as well as mixtures of cereals including sorghum, rice and barley. The qPCR assay was highly specific; the allele-specific primers did not amplify PCR products from non-target templates. It was also highly sensitive, detecting a tiny amount (>0.5\%) of waxy sorghum in the mixed samples; and it was simple and repeatable, implying the robust use of the assay.
\end{abstract}

Keywords: waxy-grain sorghum, $w x^{a}$ allele, Allele-specific primer, qPCR

\section{Background}

Waxy-grain sorghum constitutes most of the sorghum grown in Korea, reflecting its wide use in dishes such as steamed kernels with rice as well as in traditional foods ('bukkumi' - pan-fried cake, and 'tteok' - steamed or pounded cake) and beverages ('munbaeju' - distilled liquor). Non-waxy-grain sorghum is also used for human consumption (in noodles and confections), but it is mainly used for animal feed [1-3]. The waxy and hetero-waxy sorghum grain phenotypes are defined by endosperm starch

\footnotetext{
*Correspondence: cho0jm@yahoo.com

Coarse Cereal Crop Research Division, National Institute of Crop Science, Miryang, Gyeongnam 627-803, Republic of Korea
}

containing 0-15\% amylose and $85-100 \%$ amylopectin [4]. The scarcity or absence of amylose may result in higher starch and protein digestibility, which is associated with reduced kernel density and increased space between the starch granules [5]. In addition, the waxy grains were shown to have higher ethanol yield and greater conversion efficiency than the non-waxy grains, implying better quality for sorghum processing [6,7].

In sorghum (Sorghum bicolor (L.) Moench), waxy grains are caused by the loss of granule-bound starch synthase I (GBSS I) activity, leading to starch granules that contain primarily amylopectin and little or no amylose. The sorghum GBSS I gene is composed of 14 exons and small introns; exon 1 is untranslated, and exons 2-14 contain the 
coding sequence. The loss of GBSS I activity can be explained by mutations in its gene sequence; three waxy $(w x)$ mutant alleles have been identified from waxy grain sorghum lines [8] and from a Taiwanese landrace [9]. The $w x^{a}$ allele contains a large insertion in the third exon, the $w x^{b}$ allele has a missense mutation that changes glutamine 268 to a histidine, and the $w x^{c}$ allele consists of a $+1 \mathrm{G}$-to-C mutation in the $5^{\prime}$ splice site at the intron 10-exon 11 boundary $[9,10]$. To differentiate between the waxy and non-waxy alleles of the GBSS I locus, allele-specific DNA markers were developed. The $w x^{a}$-specific marker was designed based on the sequence of the insertion and amplifies a 615-bp fragment from the $w x$ allele, whereas the wildtype allele-specific primer amplifies a 523-bp fragment from the non-waxy allele. The $w x^{b}$ PCR product contains a single NcoI restriction site that can be digested to generate two DNA fragments. The wild-type PCR product, which lacks the $\mathrm{NcoI}$ site, still contains a single fragment after digestion [5]. $w x^{c}$-specific primers were designed to detect only the $w x^{c}$ allele, and the primers for the wild type detected the wild-type allele [9].

PCR-based DNA markers have been used to screen the germplasm to find new waxy alleles in accessions with waxy phenotypes but lacking known mutant alleles. By screening waxy Chinese sorghum accessions with the $w x^{a}$ and $w x^{b}$ markers, Lu et al. [11] identified two novel types of waxy mutations and developed cleaved amplified polymorphic sequence markers based on these new alleles to classify their germplasm. In this study, to determine whether a collection of Korean sorghum varieties and landraces contained any unknown types of waxy mutations, the waxy alleles in the collection were investigated using three DNA markers $\left(w x^{a}, w x^{b}\right.$ and $\left.w x^{c}\right)$. We found that all of the waxy varieties and landraces in the collection contained the $w x^{a}$ allele, implying that only this mutation was maintained during the domestic selection of Korean sorghum.

An inspection of the ingredients lists of sorghumcontaining products in Korean grocery stores shows that these products contain only waxy sorghum, but there is no way to visually determine whether any non-waxy grain is also present. PCR-based DNA markers can be used to detect the presence of waxy grain or powder in commercial products. However, neither DNA markers nor any other currently available method can be used to determine the proportion of waxy grain in a mixed-grain product. Fluorescence-based quantitative real-time PCR (qPCR) has the capacity to detect and measure minute amounts of nucleic acids in a wide range of samples from numerous sources [12,13], and it can detect and differentiate between closely related species [14,15]. Here, we report a method that uses qPCR and the waxy gene (specifically the $w x^{a}$ allele) to quantify the waxy content of various samples.

\section{Results}

waxy alleles in Korean sorghum varieties and landraces

A set of three PCR-based markers derived from three waxy alleles was synthesized to identify the $w x^{a}, w x^{b}, w x^{c}$ and wild-type GBSS I alleles in various Korean sorghum varieties and landraces. The results of genotyping using the PCR-based markers for $w x^{a}, w x^{b}$ and $w x^{c}$ should correlate with the waxy phenotype. The waxy genotype for each of the individual Korean sorghum varieties and landraces was determined via PCR for each of the three waxy allele markers. A total of 384 individuals, including 175 Korean sorghum accessions from the sorghum collection, were subjected to PCR amplification. Most of the accessions that were foreign or of unknown origin contained the wild-type allele (187 of 209 accessions [89.5\%]; 4 accessions [1.9\%] yielded no data) and the remaining 18 accessions (8.6\%) contained the $w x^{a}$ allele. Among the 175 tested Korean accessions, 150 (86\%) contained the $w x^{a}$ allele, and 25 (14\%) had no waxy alleles. One foreign accession had the $w x^{c}$ allele $\left(w x^{c} / w x^{c}\right)$. One Korean individual was heterozygous for the $w x^{c}$ allele $\left(W x / w x^{c}\right)$, and it also possessed the $w x^{a}$ allele (Figure 1 and Additional file 1). No $w x^{b}$ individuals were found in any of the accessions. This result suggested that all waxy-grained Korean sorghum was the result of one waxy allele $\left(w x^{a}\right)$ in the genome.

\section{lodine staining and amylose content of the endosperm}

To confirm the waxy allele data, the waxy and non-waxy grains of all individuals were stained with iodine $(0.2 \%$ $\left.\mathrm{I}_{2}-2 \% \mathrm{KI}\right)$, and the color of the endosperm was scored: waxy endosperm was reddish-brown, and non-waxy endosperm was dark blue. Of the 175 Korean sorghum accessions examined, iodine stained 25 of them pale or dark blue, showing that they were positive for the presence of amylose, whereas 150 accessions exhibited the waxy phenotype and stained pale or dark reddish-brown (Figure 2). All of the iodine test scores matched the waxy allele genotypes determined via DNA marker analysis, confirming that there were no new waxy alleles in the individuals that were wild type for the three waxy PCR-based markers.

To determine whether the degree of the iodine reaction was related to amylose content, the amylose content of twenty-four individuals with various reactions to the iodine solution (dark, medium, or pale blue or red) was measured. The individuals that stained blue had amylose content that ranged from 14.30 to $35.60 \%$, whereas those of the individuals that stained red ranged from 6.59 to $13.84 \%$. The accessions that stained dark blue in the iodine test contained $>21.87 \%$ amylose, with an average of 28.89\%; medium blue individuals contained $>31.02 \%$ amylose and averaged 32.13\%; and pale blue individuals contained $>23.80 \%$ amylose and averaging $27.71 \%$. By contrast, the accessions that stained dark red in the iodine test 


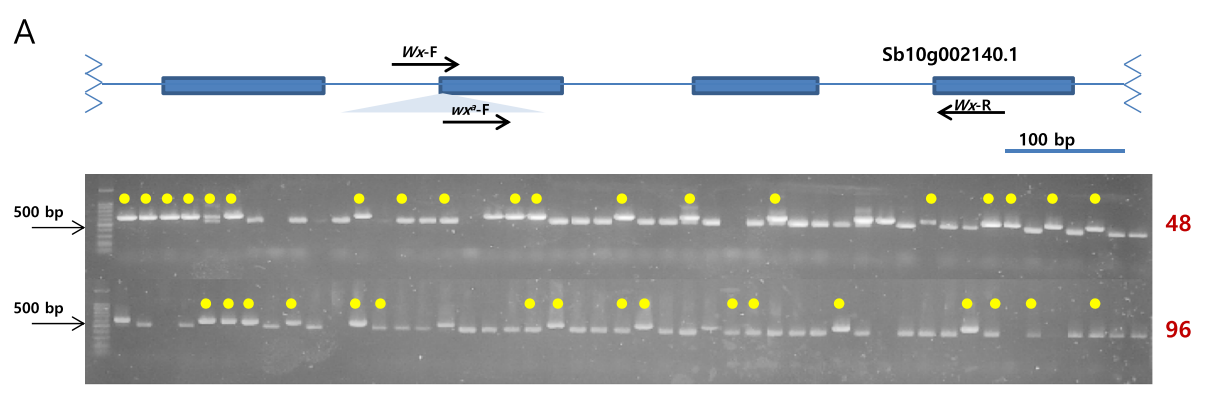

B
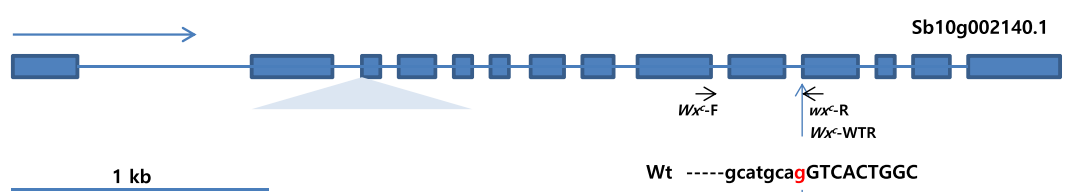

Wt -----gcatgcagGTCACTGGC

Mutation of splicing site

$w x^{c}$-----gcatgcacGTCACTGGC

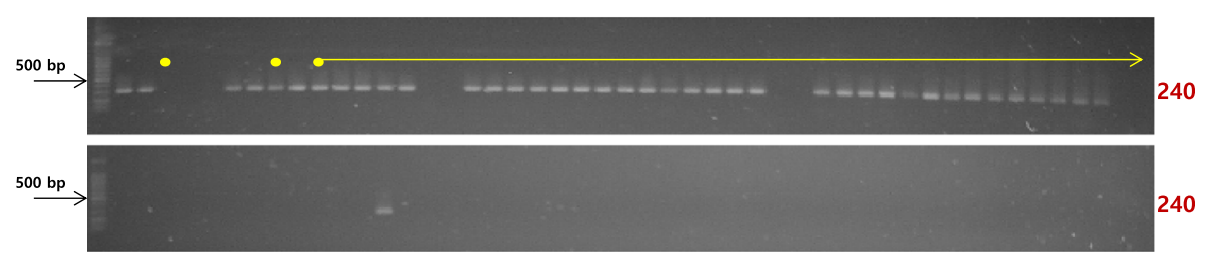

Figure 1 Genotypic analysis of Korean sorghum varieties and landraces and foreign sorghum using waxy markers. Yellow dots and arrows indicate Korean sorghum collections. Numbers in maroon indicate the identification number of the sample at the end of each electrophoregram. (A) The location of the $w x^{a}$ primer is shown on the GBSS / gene diagram. In the 3\% agarose gel, the upper band (615 bp) indicates the $w x^{a}$ mutant allele, whereas the lower band (523 bp) indicates the wild-type allele. (B) The location of the $w x^{c}$ primer is shown on the GBSS I gene diagram. The wild-type (upper panel) and $w x^{c}$ mutant alleles (lower panel) are indicated by a single 305-bp band. One individual has both a wild-type allele and a mutant $w x^{c}$ allele.

contained $<11.60 \%$ amylose and averaged $9.35 \%$; medium red individuals contained $<12.59 \%$ amylose and averaged $9.72 \%$; and pale red individuals contained $<10.49 \%$ and averaged $8.28 \%$. There were no significant differences in the amylose contents of individuals that stained the same

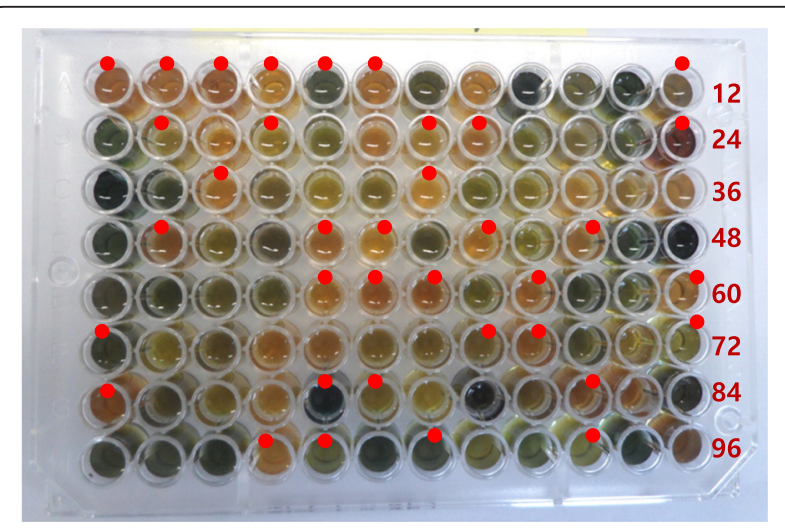

Figure 2 lodine test of Korean sorghum varieties and landraces and foreign sorghum. Red dots indicate Korean sorghum collections. The colorimetric reactions were scored as follows: red for waxy and green for non-waxy. Each well represents one independent line. Numbers in maroon indicate the identification number of the sample at the end of each row. color but to different degrees, but there was a significant difference between the blue and red groups (Figure 3), suggesting that the varied color intensity could be caused by other factors such as temperature or the presence of water-miscible organic solvents.

\section{Primer design, PCR specificity, and QPCR sensitivity}

In addition to the three allele-specific markers, eleven forward and eleven reverse primers were designed to identify the most efficient primer pairs for the qPCR-based assay. To selectively amplify products from waxy and non-waxy plants, allele-specific primers for the waxy and non-waxy alleles, as well as nonspecific primers, were designed based on a partial sequence of the GBSS I gene (starting from the end of exon 2 to the beginning of exon 4 and including part of the insertion) (Figure 4). To evaluate the PCR specificity, all possible forward and reverse primer combinations were tested on waxy and non-waxy DNA samples and on a no-template control (NTC). Among the sixteen waxy-specific combinations, the $w x^{a} 11$ forward and $w x^{a}$ 8 reverse primer pair amplified a product with stable $C_{T}$ values, and it did not produce spurious products from the non-waxy DNA samples or the NTC. By these criteria, the second and third most specific primer pairs were $w x^{a}$ 


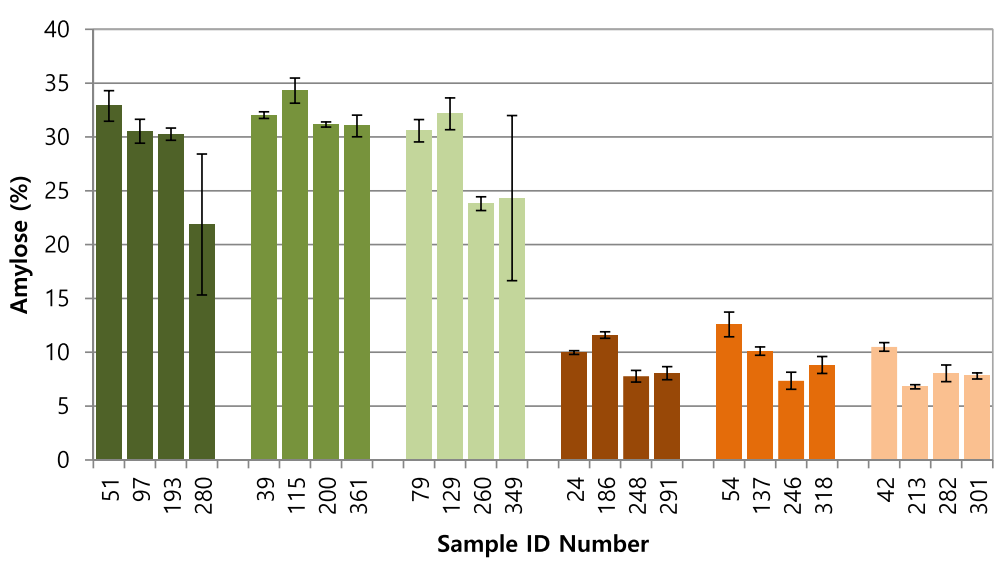

Figure 3 The amylose content of waxy and non-waxy grain samples. Based on the results of the iodine test, six sets of samples were chosen. The members of each group had similar results in the iodine test. The dark green, green and pale-green bars represent the non-waxy grains, and dark red, red and pale red bars represent the waxy grains. Each bar represents the mean amylose content as calculated from three replicate measurements. The error bars represent standard deviation.

11 forward with $w x^{a} 11$ reverse and $w x^{a} 4$ forward with $w x^{a} 8$ reverse. The other primer combinations either nonspecifically amplified the samples or did not amplify a product at all. Using the same criteria for PCR specificity, two primer pairs, the $W x$ only7 forward primer with either the Wxonly1 or Wxonly2 reverse primers, were chosen from nine non-waxy allele-specific primer combinations. Out of sixteen non-specific primer combinations, the
Wxcomn9 forward primer with either the Wxcomn 5 or Wxcomn6 reverse primers produced products from both waxy and non-waxy DNA samples but not from the NTC. Additional PCR reactions were performed and the most efficient primer pair for each sample type was determined: $w x^{a} 11$ with $w x^{a} 8$ for waxy samples, Wxonly7 with Wxonly2 for non-waxy samples and Wxcomn9 with Wxcomn6 for both types of samples.
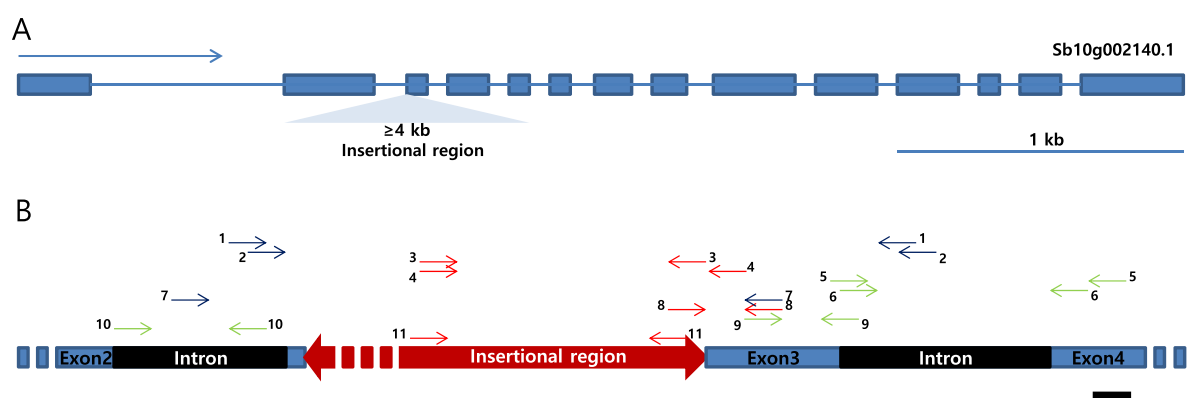

$20 \mathrm{bp}$

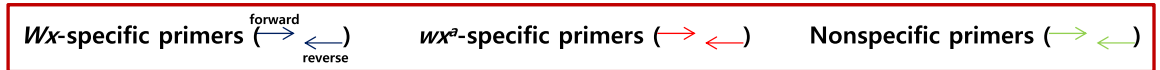

\section{C}

\begin{tabular}{|c|c|c|c|c|c|}
\hline Primer \# & Name & Group & $\begin{array}{l}\text { Forward primer sequences } \\
\left(5^{\prime}-3^{\prime}\right)\end{array}$ & $\begin{array}{l}\text { Reverse primer sequences } \\
\left(5^{\prime}-3^{\prime}\right)\end{array}$ & $\begin{array}{l}\text { Product size } \\
\text { (bp) }\end{array}$ \\
\hline 1 & Wxonly1 & $W x$-specific & GTATGTGACGTGTGTTGGGG & TGGATCTGATGGCGTGTGAT & 151 \\
\hline 2 & Wxonly2 & $W_{x}$-specific & GTGTGTTGGGGTGATGCATG & TGCACGGAAACTGGATCTGA & 153 \\
\hline 3 & wxa3 & $w x^{2}$-specific & TCAAACTCTAGACCTCATCGTGA & TGTTGAACATAATCCCGTGCA & 162 \\
\hline 4 & wxa4 & $w x^{3}$-specific & TCAAACTCTAGACCTCATCGTGA & GGGAGAGACGACCATGACAC & 195 \\
\hline 5 & Wxcomn5 & Nonspecific & CGATCACACGCCATCAGATC & ACCCGTCTCCCATCTTGATC & 196 \\
\hline 6 & Wxcomn6 & Nonspecific & GATCGATCACACGCCATCAG & AAACCTCACCGTCTCGTACC & 134 \\
\hline 7 & Wxonly7 & $W x$-specific & GGCCTGGATTCAATGTTCTT & TCCTTGTACTGGTCGTAGCG & 112 \\
\hline 8 & wxa8 & $w x^{3}$-specific & ATGCACGGGATTATGTTCAA & TCCTTGTACTGGTCGTAGCG & 65 \\
\hline 9 & Wxcomn9 & Nonspecific & CGCTACGACCAGTACAAGGA & GTGTCAGTACCTCGGACACG & 58 \\
\hline 10 & Wxcomn 10 & Nonspecific & TAAGCATTGATGCGTTCGAT & CCCAACACACGTCACATACA & 80 \\
\hline 11 & wxa11 & $w x^{2}$-specific & AGGTCAAACTCTAGACCTCATCG & ATCCCGTGCATATCTTACCC & 143 \\
\hline
\end{tabular}

Figure 4 Primer sequences and locations in the GBSS I gene containing an insertion within exon 3. (A) Schematic of the waxy gene (Sb10g002140) showing the insertion. (B) The red portion of the schematic indicates the insertion. Each short arrow designates a primer location, and the different colors represent different specificities: blue, $W x$-specific; red, $w x^{a}$-specific; and green, nonspecific. (C) The primer sequences are listed in the table. 
The primer specificity was further evaluated by amplifying DNA samples from three waxy and three non-waxy sorghum varietals using the selected primer pairs described above. The amplification plots of the waxy individuals nearly completely overlapped, and the same was true for the non-waxy individuals (Figure 5). A single, groupspecific peak was observed in the dissociation curves, ruling out the possibility of non-specific amplification. DNA fragments of the expected sizes were also confirmed through 3\% agarose gel electrophoresis.

To determine the qPCR sensitivity and detection limits using the three primer pairs, DNA samples obtained from waxy and non-waxy individual plants and grains were two-fold serially diluted from $100.0 \mathrm{ng}$ to $3.05 \mathrm{pg}$, and the samples were used to construct standard curves (Additional file 2). The linear correlation coefficients $\left(R^{2}\right)$ were calculated, and they ranged from 0.991 to 0.998 . The curves for all three primer pairs were linear over a range of $100.0 \mathrm{ng}$ to $12.21 \mathrm{pg}$, and the detection limit of all three primer pairs was approximately $12.0 \mathrm{pg}$. Below the detection limit, the plots were no longer linear due to overlapping positive signals from the DNA samples and the non-template controls $\left(C_{\mathrm{T}}\right.$ values of approximately 35$)$.

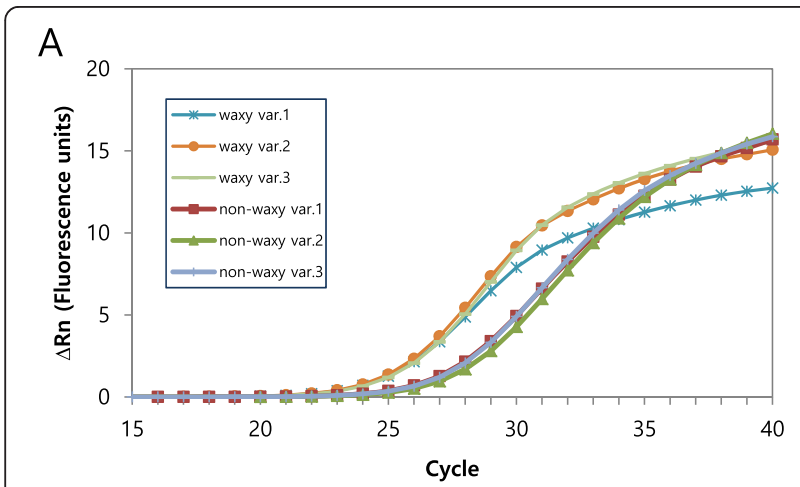

B

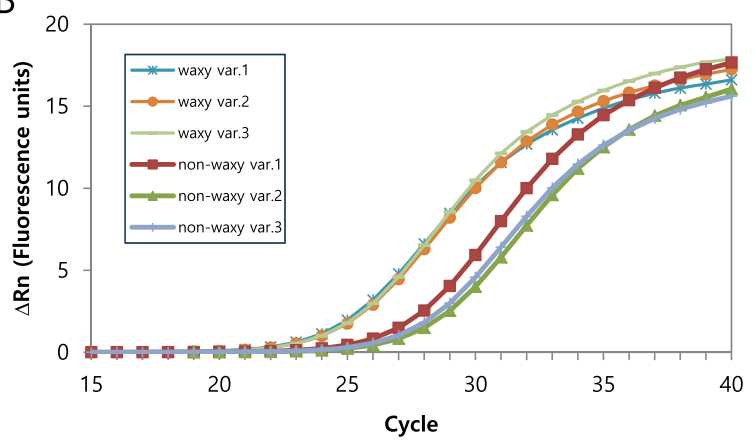

Figure 5 qPCR amplification plots of different waxy and non-waxy varieties. (A) The plots were generated via GPCR with the $w x^{a}$-specific primer pair (11 forward/8 reverse) on the waxy varieties and the $W$ xonly primer pair (7 forward/2 reverse) on the non-waxy varieties. (B) The plots were generated based on the results of $\mathrm{qPCR}$ using the nonspecific primer pair (9 forward/6 reverse) on both types of sorghum.
This phenomenon was likely due to an accumulation of primer dimers to which the SYBR Green bound. The dissociation curves for the non-specific products were different from those of the correct amplicons (results not shown). The $\mathrm{C}_{\mathrm{T}}$ values of the no-template controls overlapped with the $C_{T}$ values obtained from DNA dilutions that were below the detection limit.

\section{The detection of waxy sorghum in mixed samples}

To test the ability of the selected waxy primer pair to detect low proportions of waxy material in mixed samples containing both waxy and non-waxy sorghum powder, a set of reference mixtures of grain containing different proportions of waxy sorghum was prepared by manual grinding. DNA obtained from these reference samples was subjected to the qPCR assay, the waxy and non-waxy $C_{T}$ values in the samples were interpolated on the standard curves defined in Additional file 2, and the DNA ratios thus obtained were compared with the actual ratios of waxy and non-waxy grain present in the mixtures. The $\Delta C_{\mathrm{T}}$ was also calculated by subtracting the $\mathrm{C}_{\mathrm{TWx}}$ of the $W x$-specific primer reaction from the $\mathrm{C}_{\mathrm{T} w x}^{a}$ of the $w x^{a}$-specific primer reaction for each reference sample. The results showed a linear correlation between the actual and calculated proportions (Table 1 and Table 2). The $\Delta C_{\mathrm{T}}$ calculation allowed an estimation of the ratio of waxy versus non-waxy primer reaction products by taking the difference between $C_{\mathrm{T} w x}^{a}$ and $C_{\mathrm{T} W x}$, as in [14]. Table 1 and Table 2 show the results obtained by calculating $\Delta C_{\mathrm{T}}$, which was inversely correlated with the proportion of the waxy content of the mixtures. The $w x^{a}$-specific primers generated an unstable signal when the waxy content was less than $0.5 \%$ or when the $\Delta C_{T}$ value was negative (in the case of no signal) or greater than 10 cycles (in the case of

Table 1 The observed $C_{T}$ differences and the calculated DNA concentrations ( $N$ ) based on qPCR assays (DNA mix)

\begin{tabular}{|c|c|c|c|c|c|c|c|}
\hline \multirow{2}{*}{$\begin{array}{l}\text { \% waxy } \\
\text { DNA }\end{array}$} & \multicolumn{7}{|c|}{ DNA mix } \\
\hline & $\overline{C_{\mathrm{T}} w x^{a}}$ & $C_{\mathrm{T}} W x$ & $\begin{array}{l}\Delta C_{\mathrm{T}} w x^{a}- \\
W x\end{array}$ & $\begin{array}{l}\Delta \Delta C_{T} \\
\text { STD }\end{array}$ & $N w x^{a}$ & $N W x$ & $\begin{array}{l}\text { Calculated \% } \\
\text { waxy DNA } \\
\left(R^{2}=0.9991\right)\end{array}$ \\
\hline 100 & 24.3 & 36.0 & -11.7 & - & 4.5 & 0.0 & 99.8 \\
\hline 50 & 25.2 & 27.7 & -2.5 & 0.0 & 2.4 & 2.4 & 49.7 \\
\hline 20 & 26.7 & 26.9 & -0.2 & 0.4 & 0.9 & 4.1 & 18.2 \\
\hline 10 & 27.4 & 26.6 & 0.8 & 0.2 & 0.6 & 4.9 & 10.1 \\
\hline 5 & 28.6 & 27.2 & 1.4 & 1.1 & 0.2 & 3.4 & 6.8 \\
\hline 2 & 29.5 & 26.9 & 2.6 & 0.5 & 0.1 & 4.3 & 3.2 \\
\hline 1 & 30.6 & 26.6 & 4.0 & 0.7 & 0.1 & 5.1 & 1.3 \\
\hline 0.5 & 32.0 & 26.4 & 5.6 & 0.5 & 0.03 & 5.7 & 0.5 \\
\hline 0 & - & 26.1 & -26.1 & 0.2 & 0.0 & 7.1 & 0.0 \\
\hline
\end{tabular}

The qPCR reactions were performed using $5 \mathrm{ng}$ of DNA mix or DNA extracted from grain mix.

Mean and standard deviation (STD) values from three independent assays. 


\begin{tabular}{|c|c|c|c|c|c|c|c|}
\hline \multirow{2}{*}{$\begin{array}{l}\text { \% waxy } \\
\text { DNA }\end{array}$} & \multicolumn{7}{|c|}{ Grain mix } \\
\hline & $\overline{C_{\mathrm{T}} w x^{a}}$ & $C_{\mathrm{T}} W x$ & $\begin{array}{l}\Delta C_{\mathrm{T}} w x^{a}- \\
W x\end{array}$ & $\begin{array}{l}\Delta C_{T} \\
\text { STD }\end{array}$ & $N w x^{a}$ & $N W x$ & $\begin{array}{l}\text { Calculated \% } \\
\text { waxy DNA } \\
\left(R^{2}=0.9918\right)\end{array}$ \\
\hline 100 & 24.1 & 37.7 & -13.6 & - & 4.9 & 0.0 & 99.9 \\
\hline 50 & 24.1 & 26.1 & -2.0 & 0.0 & 5.0 & 7.2 & 40.8 \\
\hline 20 & 24.9 & 25.1 & -0.2 & 0.1 & 3.0 & 13.1 & 18.4 \\
\hline 10 & 26.2 & 25.4 & 0.8 & 0.3 & 1.3 & 11.4 & 10.1 \\
\hline 5 & 28.1 & 25.6 & 2.5 & 0.6 & 0.3 & 10.0 & 3.4 \\
\hline 2 & 28.7 & 25.6 & 3.1 & 0.2 & 0.2 & 9.7 & 2.3 \\
\hline 1 & 30.3 & 26.3 & 4.0 & 0.5 & 0.1 & 6.1 & 1.4 \\
\hline 0.5 & 29.1 & 25.2 & 3.9 & 0.1 & 0.2 & 13.1 & 1.4 \\
\hline 0 & - & 25.8 & -25.8 & 0.2 & 0.0 & 8.3 & 0.0 \\
\hline
\end{tabular}

The qPCR reactions were performed using $5 \mathrm{ng}$ of DNA extracted from each grain mixture.

The results are the means and standard deviations (STDs) of three independent assays.

primer dimers, whose $C_{T}$ value was approximately 35 ). Thus, the detection limit of the waxy allele using this qPCR method is $0.5 \%$, which is the level required to produce a stable, measurable signal from the mixed samples.

\section{The detection of waxy content in commercial samples}

The detection of waxy and non-waxy DNA in commercial cereal products can be further complicated by the presence of various cereals, and the PCR reactions could be hindered by the presence of inhibitors in the DNA extract. To test the behavior of the qPCR system and the selected primer pairs on commercial samples, PCR reactions were run on nine different commercial sorghum brands: seven sorghum grain products containing only waxy grains and two sorghum powder products with unknown sorghum content declared on the package label. DNA was extracted from all of the products, and the amplification of waxy DNA was tested using the three selected primer pairs. The reactions for products 1,2 , and 6 through 8 suggested no or $<0.5 \%$ non-waxy grain in these products. Product 3 generated products for both waxy and non-waxy grains, and product 9 generated a PCR product only with the non-waxy primers (Figure 6). To determine whether the PCR reactions were inhibited in the samples obtained from the commercial grains and powders, the reactions were compared with control reactions using waxy (10 in Figure 6) and non-waxy (11 in Figure 6) DNA. No inhibition was observed in these tests, suggesting that the results from the commercial grain and powder products corresponded to the amplifiable DNA present in the extracts. It is worth noting that the qPCR assay and primer pairs suggested the presence of non-waxy grains in product 3. Because the $12.0 \mathrm{pg}$ detection limit for waxy and non-waxy DNA was determined by standard curve analysis, it is reasonable to hypothesize that grain samples containing $>0.5 \%$ waxy DNA would yield a positive signal from the $w x^{a}$-specific primer pair in reactions containing 5 ng of total grain DNA regardless of the waxy DNA content. Based on the $\Delta C_{T}$ value (negative 2.27), the sorghum content of product 3 was approximately $50 \%$ non-waxy.

\section{Discussion}

A rapid and sensitive qPCR-based method to detect waxy sorghum DNA was developed to provide stakeholders with a tool to evaluate the quality of sorghum products. To the best of our knowledge, tools to quantify waxygrain sorghum material have not been developed to date. Therefore, we have developed a technique to detect and quantify the amount of waxy sorghum DNA in a mixed sample. Our method could be applied to calculate the ratio of waxy to non-waxy materials, which would be particularly informative for cereal processing. Thus, the widespread application of qPCR analysis to quantify waxy sorghum DNA is needed for sorghum processing and quality control.

The waxy sorghum genotypes can be classified based on three mutant alleles, which can be detected using specific DNA markers that are based on their sequences [5,9]. The Korean sorghum germplasm includes more than 1600 landraces that were collected from all of the counties in South Korea and are maintained at the RDA Genebank. In this study, we sampled approximately $10 \%$ of all catalogued Korean landraces and analyzed the frequency of the waxy alleles using three mutant allele-specific DNA markers. Each individual was a randomly selected representative of each city or county in the nation. Because Korean sorghum landraces have narrow gene pools, as shown by genetic diversity analysis with SSR markers (data not shown, manuscript in preparation), one random sample per city or county should be sufficient to investigate the waxy alleles. Six varieties of Korean sorghum breeding lines were also included in this study because they are the only widely distributed sorghum germplasm in the country. All tested Korean landraces and breeding materials that had waxy genotypes contained the $w x^{a}$ allele, and one of these $w x^{a}$ landraces was heterozygous for the $w x^{c}$ allele. There was no indication of the existence of a new allele in the Korean sorghum germplasm and breeding materials because the genotypic and phenotypic data (determined by iodine staining and amylose content) matched perfectly. Thus, we concluded that the $w x^{a}$ allele could be a target for the detection and quantification of the waxy content in mixed samples.

Optimization of the qPCR assay was critical to its successful use in the detection of waxy materials. Both the primer and the target sequences can affect the efficiency, specificity and accuracy of PCR assays [16]. We designed 


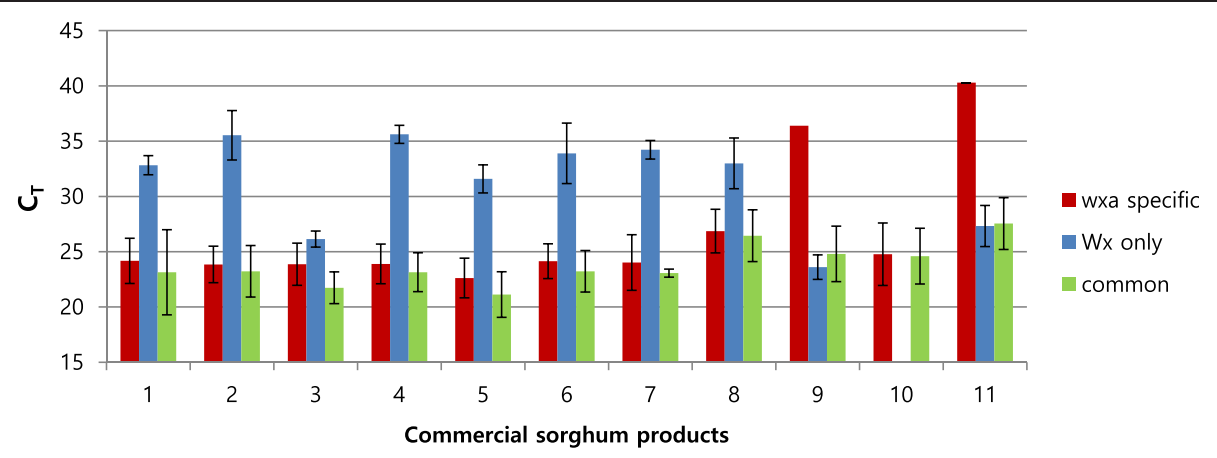

Figure 6 qPCR assay on commercial sorghum products. Numbers 1 through 9 indicate commercial sorghum products. Numbers 10 and 11 represent control waxy and non-waxy DNA, respectively. Each bar represents the mean $C_{T}$ value calculated from three replicate measurements. The missing bar indicates that no signal was detected. The error bars indicate standard deviation.

novel sets of primers based on three different allelespecific DNA markers from the region flanking the insertion in the GBSS I gene. To provide ideal qPCR assay conditions, the primers were designed to amplify small ( $<200$ bp) PCR products, as per the guidelines of [17]. Out of all possible primer pair combinations, the selected primer pairs showed good specificity in each type of sorghum plant; the waxy-specific primers did not amplify products from non-waxy sorghum plants and vice versa. The nonspecific primer pair amplified products from both types of sorghum. Moreover, standard curves constructed using different waxy and non-waxy sorghum varieties showed similar amplification efficiencies, further supporting the specificity of the primers in calculating the proportion of waxy DNA. It should be mentioned that BLAST analysis of the primer sequences generated hits against only the sorghum GBSS I gene, and the analysis showed $100 \%$ identity between the sequences of the GBSS I gene and the primers.

The sensitivity (detection limit) of the qPCR assay can be used as another criterion to indicate its robustness. Standard curves generated using DNA from waxy sorghum, nonwaxy sorghum, and mixtures of both demonstrated that our qPCR analysis could efficiently measure as little as $12.0 \mathrm{pg}$ of DNA. The amplification cycle of non-waxy sorghum was one cycle greater than that of waxy sorghum, and the difference in $C_{T}$ values between the waxy and non-waxy PCR products might be attributed to the characteristic curves of the samples' amplicons. The presence of various PCR inhibitors, such as polyphenols, tannins and polysaccharides, may reduce the sensitivity of the qPCR assay. However, this was not the case in this study. We constructed standard curves by diluting DNA extracted with a commercial kit, and we did not detect a significant difference between the waxy and non-waxy samples. The numerous replicates resulted in similar standard curves, demonstrating that the assay is reproducible and highly robust.
The present waxy qPCR assay could also be used to determine the proportions of waxy and non-waxy materials in mixed products. After using reference samples to generate standard curves, the absolute quantitative values of waxy and non-waxy DNA could be calculated, and the difference in $C_{T}$ values could be calculated to estimate their proportions in a mixture, as shown in the results. The proportion of waxy DNA in a mixed sample could be determined by calculating the cycle differences in the qPCR assays using waxy- and non-waxy-specific primers without further calculation. This calculation should be based on the careful measurement of individual samples, but the $\Delta C_{\mathrm{T}}$, which can be easily acquired, may be acceptable for most practical applications. This quantitative approach may allow for the detection of very low levels of waxy DNA in a sample without first determining the DNA concentration.

waxy mutants that affect the function of GBSS have been identified in several small-grain crops such as wheat [18], rice [19,20], barley [21,22], corn [23] and millet [24]; the structures of the non-waxy alleles in these cereal species are very similar, but the waxy mutant alleles in each species are caused by a variety of mutations such as a point mutations, deletions and the insertion of transposable elements. Because of the structural similarity between target sequences, mutant alleles with deletions or insertions could be analyzed in a manner similar to that used for sorghum. Provided that the target polymorphism was previously validated via sequencing, this assay method could be applied to species for which a large number of sub-species, varieties or hybrids are present in the market.

\section{Conclusions}

This study demonstrates that the presence and quantity of waxy-grain sorghum or waxy-grain sorghum powder in mixed cereal products can be evaluated using quantitative 
real-time PCR with waxy allele-specific primers. Three different primer pairs were used, and each pair specifically amplified a product from only plants containing the target allele. This specificity enabled us to identify waxy and non-waxy genotypes and estimate their proportions in mixed samples containing both waxy and non-waxy sorghum grains or powder. Our method could be applied to other cereal crops that have similar waxy mutant alleles using primers that are similar to those used in this study. This method also provides a diagnostic tool to measure the waxy sorghum content declared on the labels of commercial cereal products.

\section{Methods}

\section{Plant materials and DNA extraction}

Seeds of the Korean sorghum landraces were obtained from the Rural Development Administration (RDA) Genebank Information Center (Suwon, Rep. of Korea), and the Korean sorghum varieties were acquired from the National Institute of Crop Science (Miryang, Rep. of Korea). Genomic DNA from all sorghum accessions was extracted from two- to three-week-old leaves, and for the commercial sorghum products, DNA was extracted from the sorghum grains or powder. All DNA extraction procedures were conducted using a DNeasy Plant Mini kit (QIAGEN, Valencia, California, USA) according to the manufacturer's instructions.

\section{PCR analysis of the three waxy alleles}

Three waxy allele markers were designed based on DNA markers used in previous articles [5,9]; the PCR primers used to detect $w x^{a}, w x^{b}$ and $w x^{c}$ were $w x^{a}$ F, 5'-CGTGGCGAGATCAAACTCTA-3'; Wx-F, 5' GGCCTGGATTCAATGTTCTT-3'; $W x-\mathrm{R}$ (which can also be used as $\left.w x^{a}-\mathrm{R}\right), 5^{\prime}$-GCAGCTGGTTGTCCT TGTAG-3'; $w x^{b}-\mathrm{F}, 5^{\prime}$-CGACCGTGTGTTCATTGAC CAC-3'; $w x^{b}-\mathrm{R}, 5^{\prime}$-TTGTTCAGTGCCTTTGCCTCG$3^{\prime} ; w x^{c}$-F, GCTGGTTCTGAGTGCAACAA; $w x^{c} \mathrm{WT}-\mathrm{R}$, 5'-ACTTCTTCTTGCCAGTGACC-3' (for the wild type); and $w x^{c}-\mathrm{R}, 5^{\prime}$-ACTTCTTCTTGCCAGTGACG$3^{\prime}$ (for $w x^{c}$ ). The PCR reactions were performed in a final volume of $10 \mu \mathrm{l}$ and contained $1 \mu \mathrm{l}(5 \mathrm{ng})$ of DNA template, primers $(0.5 \mu \mathrm{M}$ each), $25 \mathrm{nM}$ dNTPs, $10 \mathrm{X}$ buffer with $\mathrm{MgCl}_{2}$, and $2.5 \mathrm{U}$ of Taq DNA polymerase (iNtRON Biotechnology, Seongnam-si, Rep. of Korea). The PCR conditions were an initial denaturation at $95^{\circ} \mathrm{C}$ for $5 \mathrm{~min} ; 40$ cycles of denaturation at $95^{\circ} \mathrm{C}$ for $20 \mathrm{~s}$, annealing at $60^{\circ} \mathrm{C}$ for $30 \mathrm{~s}$, and extension at $72^{\circ} \mathrm{C}$ for $50 \mathrm{~s}$; and a final extension at $72^{\circ} \mathrm{C}$ for $7 \mathrm{~min}$. All PCR amplifications were performed in a GeneAmp PCR System 9700 (Applied Biosystems, Foster City, CA). The PCR products were analyzed by $3 \%$ (wt/vol) agarose gel in $1 \mathrm{X}$ TBE electrophoresis buffer containing $0.1 \mathrm{ppm}$ RedSafe ${ }^{\mathrm{me}}$ Nucleic Acid Staining Solution (Chembio Ltd., Hertfordshire,
England). SiZer" -100 DNA Marker Solution (iNtRON Biotechnology, Seongnam-si, Rep. of Korea) was used as the DNA size marker.

\section{lodine test (I2-KI staining)}

Seeds from self-fertilized individuals from the indicated sorghum accessions were cracked and stained with iodine solution $\left(0.2 \% \mathrm{I}_{2}-2 \% \mathrm{KI}\right)$. Endosperm that was reddish-brown or dark blue in color was scored as waxy or non-waxy, respectively [25].

\section{Apparent amylose content}

Sorghum grains $(100 \mathrm{mg})$ were milled in $1 \mathrm{ml}$ of ethanol, shaken for $15 \mathrm{~min}$ and incubated with $9 \mathrm{ml}$ of $1 \mathrm{~N}$ $\mathrm{NaOH}$ for $20 \mathrm{~min}$ at $95^{\circ} \mathrm{C}$. Five $\mathrm{ml}$ of the mix was transferred to a $100-\mathrm{ml}$ flask and incubated with $1 \mathrm{ml}$ of $1 \mathrm{~N}$ $\mathrm{CH}_{3} \mathrm{COOH}$ and $2 \mathrm{ml}$ of iodine solution (2\% KI solution + $0.2 \%$ iodine) for $30 \mathrm{~min}$ at $30^{\circ} \mathrm{C}$. The apparent amylose content of the solution was measured at $620 \mathrm{~nm}$ using a UV-2450 (Shimadzu Co., Kyoto, Japan); this technique is based on the colorimetric measurement of the iodinestarch complex (absorbance at $620 \mathrm{~nm}$ ) as described previously [26]. The 'M 162', 'Dongjin' and 'Koami' rice cultivars were used as controls. The starch from the sorghum grains was analyzed in triplicate.

\section{qPCR primer design}

Three primers were designed based on the sequences flanking the insertion in the third exon of the sorghum GBSS I gene: non-waxy-specific ( $W x$ only), waxy-specific $\left(w x^{a}\right)$ and non-specific (Wxcomn). The $W x$ only primers were designed to only amplify a PCR product from the DNA of non-waxy plants. Because the $w x^{a}$ primers were designed based on the insertion sequence, they can be used for only waxy plants. The $162 \mathrm{bp}$ at the 3 ' end of the insertion, which were used to design the waxy-specific primers, were determined by sequencing the PCR product amplified by the $w x^{a}$ primers [5]. The $W x$ comn primers, which amplified DNA from both waxy and non-waxy plants, were designed based on the GBSS I sequence located $83 \mathrm{bp}$ downstream of the insertion. Primer design was performed using Primer3web version 4.0.0 (http://primer3.ut.ee/) with the $\min$ and $\max$ primer $\mathrm{Tm}$ changed to 58 and $60^{\circ} \mathrm{C}$, respectively. Information on all eleven primer pairs is listed in Figure 4. A BLAST search (Basic Local Alignment Search Tool, http:// blast.ncbi.nlm.nih.gov/Blast.cgi) was conducted to estimate the specificity of each primer. Real-time PCR amplification was performed with an Applied Biosystems 7300 Real Time PCR System (Foster City, California, USA).

qPCR conditions and test of amplification efficiency qPCR was performed using an Applied Biosystems 7300 Real Time PCR System in an optical 96-well plate 
covered with optical sealing tape (Applied Biosystems, Foster City, California, USA). Polymerase chain reactions were carried out in a final volume of $20 \mu \mathrm{l}$, which contained $10 \mu \mathrm{l}$ of SYBR Select Master Mix (Applied Biosystems, Foster City, California, USA), $5 \mathrm{ng}$ of template DNA and primers $(0.4 \mu \mathrm{M}$ each). In addition, to detect genomic DNA contamination, control PCRs with no template (NTC) were also prepared for each primer pair. The reaction mixtures were incubated for a $10 \mathrm{~min}$ initial denaturation at $95^{\circ} \mathrm{C}$, followed by $40 \mathrm{amp}-$ lification cycles of $15 \mathrm{~s}$ at $95^{\circ} \mathrm{C}$ and $60 \mathrm{~s}$ at $60^{\circ} \mathrm{C}$. All reactions were performed in duplicate for each template DNA sample. Following qPCR amplification, to confirm the presence of a single product and the absence of primer dimers, melting curve analysis from 55 to $95^{\circ} \mathrm{C}$ was conducted with a temperature gradient of $0.5^{\circ} \mathrm{C}$ every $10 \mathrm{~s}$. The presence of a single qPCR product was further verified by $3 \%$ agarose gel electrophoresis.

\section{Standard curves}

Standard curves were generated by plotting the cycle threshold $\left(\mathrm{C}_{\mathrm{T}}\right)$ values of the qPCR reactions from serially diluted DNA. Two different genomic DNA samples (waxy and non-waxy) were two-fold serially diluted, and the dilutions were used to construct standard curves for the $w x^{a}, W x$ only and $W x$ comn primer pairs. The $C_{\mathrm{T}}$ value for each dilution was plotted against the log of the template DNA concentration. The qPCR efficiency and regression coefficient $\left(R^{2}\right)$ for each primer pair were acquired via the standard curves generated using this method.

\section{Quantification of mixed cereal grain samples}

Mixed grain samples were prepared using various proportions of powders from waxy and non-waxy sorghum grains $(0,0.5,1,2,5,10,20,50$, or $100 \%$ waxy grains, with the remainder from non-waxy grains). The samples were prepared by weighing the grains in the given ratio, evenly grinding with a mortar and pestle and extracting DNA. Mixed DNA samples were also prepared by directly combining DNA from waxy and non-waxy sorghum in the same ratios as mixed grain powder samples. Commercial cereal products were purchased from the grocery store and used for DNA extraction to confirm the applicability of the qPCR method developed in this study.

The proportion of waxy sorghum in mixtures of waxy and non-waxy sorghum was calculated as follows: the slopes and intercepts of the standard curves described above were calculated for both the waxy and non-waxy samples. qPCR amplification of DNA from the cereal mixtures was carried out in triplicate, and the mean $C_{T}$ values were interpolated in the corresponding standard curve to obtain the DNA content of the sample. Then, the ratio of the samples in the mixture was calculated as

$$
\begin{aligned}
\% \text { waxy }= & 100 \times[\text { Waxy DNA content } / \\
& (\text { Waxy DNA }+ \text { non-waxy DNA })]
\end{aligned}
$$

\section{Additional files}

Additional file 1: List of Korean sorghum varieties and landraces and foreign sorghum varieties used in this study.

Additional file 2: Standard curves derived from qPCR using primer pairs specific for waxy and non-waxy genomic DNA or that were nonspecific (amplified both varieties). For each genomic DNA sample, 100.0 ng of DNA was serially diluted two-fold and amplified using a specific primer pair, and the results were used to draw a standard curve. Three symbols-one for each replicate-are displayed at each DNA concentration. The correlation coefficients were calculated to verify the reliability and efficiency of the qPCR reactions. (A) The results of using the Wxcomn primer pair on non-waxy sorghum DNA, (B) the results of using the Wxcomn primer pair on waxy sorghum DNA, (C) the results of using the $w x^{a}$-specific primer pair on waxy sorghum DNA, and (D) the results of using the Wxonly primer pair on non-waxy sorghum DNA.

\section{Abbreviations}

GBSS I: Granule-Bound Starch Synthase I; qPCR: Quantitative real-time PCR; NTC: Non-template control; C.: Cycle threshold.

\section{Competing interests}

The authors declare that they have no competing interests.

\section{Authors' contributions}

$J C$ conceived of the manuscript and designed all experiments, performed the experiments, analyzed the data, and drafted the manuscript. TJ and JK conceived of the manuscript, performed experiments and analyzed the data. $\mathrm{SS}, \mathrm{JK}, \mathrm{KW}, \mathrm{JL}$ and $\mathrm{MC}$ performed experiments and analyzed the data. $\mathrm{IO}$ conceived of the manuscript. All authors read and approved the manuscript.

\section{Acknowledgements}

This work was carried out with the support of "Cooperative Research Program for Agriculture Science \& Technology Development (PJ00910102)" Rural Development Administration, Republic of Korea.

Received: 6 November 2014 Accepted: 2 March 2015

Published online: 19 March 2015

\section{References}

1. Kim HY, Seo HI, Ko JY, Song SB, Kim JI, Lee JS, et al. Physicochemical characteristics of the muffin added glutinous and non-glutinous sorghum (Sorghum bicolor L. Moench) powder. Korean J Food Nutr. 2012;25(3):490-8.

2. Ko JY, Woo KS, Kim JI, Song SB, Lee JS, Kim HY, et al. Effects of quality characteristics and antioxidant activities of dry noodles with added sorghum flour by characteristics of endosperm. J Korean Soc Food Sci Nutr. 2013;42(8):1227-35.

3. Woo KS, Ko JY, Kim Jl, Lee JS, Song SB, Cho J, et al. Cooking properties and antioxidant activity of cooked rice according to the addition of glutinous and non-glutinous sorghum. Korean J Crop Sci. 2013;58(4):399-407.

4. Rooney LW, Serna-Saldivar SO. Sorghum. 2nd ed. New York: Marcel Dekker; 2000.

5. Sattler SE, Singh J, Haas EJ, Guo L, Sarath G, Pedersen JF. Two distinct waxy alleles impact the granule-bound starch synthase in sorghum. Mol Breed. 2009;24(4):349-59.

6. Wang D, Bean S, McLaren J, Seib P, Madl R, Tuinstra M, et al. Grain sorghum is a viable feedstock for ethanol production. J Ind Microbiol Biotechnol. 2008;35(5):313-20.

7. Yan S, Wu X, Bean SR, Pedersen JF, Tesso T, Chen YR, et al. Evaluation of waxy grain sorghum for ethanol production. Cereal Chem. 2011;88(6):589-95. 
8. Pedersen JF, Bean SR, Graybosch RA, Park SH, Tilley M. Characterization of waxy grain sorghum lines in relation to granule-bound starch synthase. Euphytica. 2005;144(1-2):151-6.

9. Kawahigashi H, Oshima M, Nishikawa T, Okuizumi H, Kasuga S, Yonemaru J-i, et al. A novel waxy allele in sorghum landraces in East Asia. Plant Breed. 2013;132(3):305-10.

10. McIntyre CL, Drenth J, Gonzalez N, Henzell RG, Jordan DR. Molecular characterization of the waxy locus in sorghum. Genome. 2008;51 (7):524-33.

11. Lu Y, Zhao G, Li Y, Fan J, Ding G, Zhao J, et al. Identification of two novel waxy alleles and development of their molecular markers in sorghum. Genome. 2013;56(5):283-8.

12. Hierro N, Esteve-Zarzoso B, Gonzalez A, Mas A, Guillamon JM. Real-time quantitative PCR (QPCR) and reverse transcription-QPCR for detection and enumeration of total yeasts in wine. Appl Environ Microbiol. 2006;72(11):7148-55.

13. Johansen P, Vindelov J, Arneborg N, Brockmann E. Development of quantitative PCR and metagenomics-based approaches for strain quantification of a defined mixed-strain starter culture. Syst Appl Microbiol. 2014;37(3):186-93.

14. Aldeguer M, Lopez-Andreo M, Gabaldon JA, Puyet A. Detection of mandarin in orange juice by single-nucleotide polymorphism qPCR assay. Food Chem. 2014;145:1086-91.

15. Sandberg M, Lundberg L, Ferm M, Malmheden YI. Real time PCR for the detection and discrimination of cereal contamination in gluten free foods. Eur Food Res Technol. 2003;217(4):344-9.

16. Bustin SA, Benes V, Garson JA, Hellemans J, Huggett J, Kubista M, et al. The MIQE guidelines: minimum information for publication of quantitative real-time PCR experiments. Clin Chem. 2009;55(4):611-22.

17. Thornton B, Basu C. Real-time PCR ( $(P C R)$ primer design using free online software. Biochem Mol Biol Educ. 2011;39(2):145-54.

18. Nakamura T, Yamamori M, Hirano H, Hidaka S, Nagamine T. Production of waxy (amylose-free) wheats. Mol Gen Genet. 1995;248(3):253-9.

19. Isshiki M, Morino K, Nakajima M, Okagaki RJ, Wessler SR, lzawa T, et al. A naturally occurring functional allele of the rice waxy locus has a GT to $\pi$ mutation at the $5^{\prime}$ splice site of the first intron. Plant J. 1998;15(1):133-8.

20. Larkin PD, Park WD. Transcript accumulation and utilization of alternate and non-consensus splice sites in rice granule-bound starch synthase are temperature-sensitive and controlled by a single-nucleotide polymorphism. Plant Mol Biol. 1999;40(4):719-27.

21. Hylton C, Denyer K, Keeling P, Chang M-T, Smith A. The effect of waxy mutations on the granule-bound starch synthases of barley and maize endosperms. Planta. 1996;198(2):230-7.

22. Taira T, Fujita N, Takaoka K, Uematsu M, Wadano A, Kozaki S, et al. Variation in the primary structure of waxy proteins (granule-bound starch synthase) in diploid cereals. Biochem Genet. 1995;33(7-8):269-81.

23. Shure $M$, Wessler $S$, Fedoroff $N$. Molecular identification and isolation of the Waxy locus in maize. Cell. 1983;35(1):225-33.

24. Hachiken T, Sato K, Hasegawa T, Ichitani K, Kawase M, Fukunaga K. Geographic distribution of Waxy gene SNPs and indels in foxtail millet, Setaria italica (L.). P Beauv Genet Resour Crop Evol. 2012;60(4):1559-70.

25. Pedersen JF, Bean SR, Funnell DL, Graybosch RA. Rapid iodine staining techniques for identifying the waxy phenotype in sorghum grain and waxy genotype in sorghum pollen. Crop Sci. 2004;44(3):764-7.

26. Juliano BO. A simplified assay for milled rice amylose. Cereal Sci Today 1971;16:334-40.

\section{Submit your next manuscript to BioMed Central and take full advantage of:}

- Convenient online submission

- Thorough peer review

- No space constraints or color figure charges

- Immediate publication on acceptance

- Inclusion in PubMed, CAS, Scopus and Google Scholar

- Research which is freely available for redistribution 